\title{
Saúde Sexual de Mulheres que Fazem Sexo com Mulheres: Prevenção de Infecções Sexualmente Transmissíveis
}

\author{
Giulliane Ramos Londero, ${ }^{1}$ Melissa Medeiros Braz ${ }^{2}$
}

\section{RESUMO}

Objetivo: Investigar o uso de métodos preventivos de infecções sexualmente transmissíveis (ISTs) em mulheres jovens que fazem sexo com outras mulheres. Métodos: Pesquisa observacional, de caráter transversal com abordagem quantitativa, realizada entre os meses de janeiro e abril de 2018. A amostra foi composta por mulheres com idade entre 18 e 29 anos. Para a coleta de dados foi utilizado um questionário adaptado com quatro domínios: identificação, história ginecológica, conhecimento sobre o contágio por ISTs e informações sobre métodos de prevenção de ISTs. Resultados: A maioria das entrevistadas (54,5\%) relata conhecer algum método de prevenção de ISTs para o sexo entre mulheres, mas não fazem o uso destes em suas relações sexuais. Apenas 8,9\% utiliza algum método de barreira nas relações sexuais que não envolvem o uso de acessórios sexuais e $9,8 \%$ nas que envolvem. Conclusão: As mulheres que fazem sexo com mulheres (MSM) têm conhecimento sobre os riscos de contágio por ISTs a que se expõem ao realizar sexo sem proteção, porém, ainda sim não têm o costume de usar métodos preventivos. Os profissionais de saúde não consideram os riscos de contágio a que as MSM se expõem e não as informam sobre como se prevenir adequadamente e a importância de adotar os métodos de barreira como prática cotidiana.

Palavras-chave: bissexualidade; homossexualidade feminina; saúde sexual; sexo sem proteção.

\section{SEXUAL HEALTH OF WOMEN WHO HAVE SEX WITH WOMEN: PREVENTION OF SEXUALLY TRANSMITTED INFECTIONS ABSTRACT}

Objective: The survey's aim is to investigate the use of sexually transmitted infections (STIs) preventive methods by young women who have sex with other women. Methods: Observational research, using cross-sectional and quantitative approach, performed between January and April of 2018. The sample was composed of women aged between 18 and 29 years. To collect all the data, na adapted questionnaire with four fields was used: identification, gynecologic history, personal knowledge about STI contamination and the access to information about sexually transmitted infections preventive methods. Results: The majority of the interviewed $(54,5 \%)$ report to know some STI prevention methods in sexual relations between women, but don't use them in their sexual relations. Only 8,9\% use some barrier method in their sexual relations without sexual accessories, and $9,8 \%$ use some method when there are sexual accessories involved. Conclusion: Women who have sex with women (WSW) know about the STIs contagion risks they are exposed to when having unprotected sex, but still don't have the habit of using preventive methods. Health professional don't consider them as a contagion risk group and don't give them information about how to properly protect themselves and about the importance of embracing the barrier methods as a daily practice.

Keywords: bisexuality; homosexuality female; sexual health; unsafe sex.

RECEBIDO EM: 8/8/2020

MODIFICAÇÕES SOLICITADAS EM: 13/1/2021

ACEITO EM: 20/2/2021

\footnotetext{
${ }^{1}$ Autora correspondente. Universidade Federal de Santa Maria (UFSM). Av. Roraima no 1000 - Cidade Universitária, Bairro Camobi. Santa Maria/RS, Brasil. CEP 97105-900. http://lattes.cnpq.br/2893708270152523 https://orcid.org/0000-00034093-0272. giullianelondero@gmail.com

2 Universidade Federal de Santa Maria (UFSM). Santa Maria/RS, Brasil.
} 


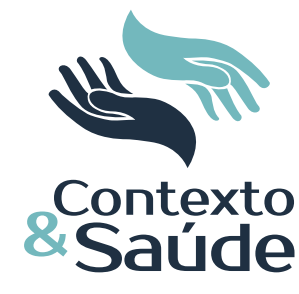

\section{INTRODUÇÃO}

O termo "mulheres que fazem sexo com outras mulheres" (MSM) refere-se a qualquer mulher que pratique relações sexuais com outra mulher (ROWEN et al., 2013). Até pouco tempo acreditava-se que existia baixo risco de transmissão de infecções sexualmente transmissíveis (ISTs) no sexo entre mulheres, por isso a saúde sexual desse grupo específico era pouco estudada (LOGIE, 2015). Agora, porém, é sabido que MSM estão envolvidas em práticas sexuais de risco que podem as predispor ao contágio por ISTs (TAT; MARRAZZO; GRAHAM, 2015).

Em razão da existência de poucas intervenções de saúde pública específicas para MSM, é proporcionada a esse grupo uma pequena quantidade de informações sobre os métodos preventivos adequados e a sua efetividade (DALY; SPICER; WILLAN, 2016). Na literatura o conhecimento descrito sobre práticas sexuais de MSM e fatores de riscos para IST é precário (FUJII, 2019), e a invisibilidade desse grupo dificulta a realização de intervenções que visem à prevenção, diagnóstico e tratamento (RUFINO et al., 2018).

Os profissionais da saúde pressupõem a heterossexualidade das mulheres já oferecendo métodos de prevenção masculinos e anticoncepcionais hormonais orais, sem considerarem que a paciente possa ser ativa sexualmente com outras mulheres (SOUTO PEREIRA; SWAINSTON; BECKER, 2019). Há mulheres que não discutem sua orientação sexual com o médico ginecologista por receio de sofrerem discriminação ou por acharem essa informação irrelevante para o atendimento. Em contrapartida, há mulheres que apenas relatam sua orientação por precisarem de cuidados específicos (ZAIDI et al., 2016).

Observa-se que, quando se trata do direito à saúde das MSM, existem muitas incertezas e invisibilidades. Por ainda ser considerado assunto tabu, pouco discutido e não falado abertamente, leva à precária educação sexual e educação em saúde. A falta de conhecimento e de preparo adequado dos profissionais da saúde para lidar com essas mulheres, associada aos mitos envolvendo as relações sexuais entre mulheres, gera uma menor adesão aos métodos preventivos de ISTs e, por consequência, uma maior exposição a essas doenças.

Mesmo sendo necessário que os profissionais da saúde entendam as MSM como uma população-alvo para a prevenção de ISTs, são raras as discussões sobre práticas sexuais seguras com mulheres lésbicas e bissexuais (FUJII, 2019). A ausência de informações sobre saúde e sexualidade é um dos principais motivos que deixa as MSM em situações vulneráveis (OLIVEIRA; NERY, 2016).

Estudos nessa área são recentes e escassos, e grande parte realizados fora do Brasil (DALY; SPICER; WILLAN, 2016; LOGIE et al., 2015; LOGIE, 2015; MUZNY et al., 2013a; MUZNY et al., 2013b). Por isso, pesquisas relacionadas à prevenção de ISTs em mulheres brasileiras que fazem sexo com outras mulheres podem ser úteis para diminuir os números de infecções no país, além de disseminar o conhecimento sobre essa pauta e garantir, para uma minoria, o seu direito à saúde. Com isso, este estudo visa a investigar o uso de métodos preventivos de ISTs em mulheres jovens que fazem sexo com outras mulheres.

Editora Unijuí - Revista Contexto \& Saúde - ISSN 2176-7114 - v. 21, n. 43, jul./set. 2021 


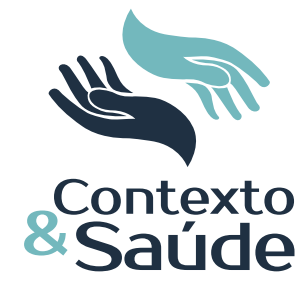

\section{MÉTODOS}

Trata-se de um estudo observacional, de abordagem quantitativa e caráter transversal, com mulheres jovens que fazem sexo com outras mulheres, no período de janeiro a abril de 2018. Para compor a amostra utilizou-se o método de estimação por proporção, por intermédio dos dados do estudo piloto realizado com 40 participantes por meio da análise dos questionários respondidos. Obteve-se uma proporção de uso de método preventivo de 7,3\%. A precisão absoluta utilizada foi de $10 \%$ e o nível de significância de $5 \%$. A amostra do estudo é definida como não probabilística e o meio de pesquisa foi o método bola de neve, muito frequente nas pesquisas que envolvem MSM (TAT; MARRAZZO; GRAHAM, 2015), pois é utilizado para grupos difíceis de serem acessados, em que se espera que uma participante convide outra, que acaba indicando outra e assim, sucessivamente, (VARELA et al., 2017).

Foram incluídas mulheres que já tiveram relação sexual com outras muIheres e mulheres que se definiram como lésbicas ou bissexuais com idade entre 18 e 29 anos, conforme o limite definido para mulheres jovens (BRASIL, 2013). Foram excluídas mulheres transgêneros e transexuais, por entender que é uma população com especificidades e necessidades de cuidado diferentes do grupo de MSM (TAT; MARRAZZO; GRAHAM, 2015), e mulheres que não responderam corretamente o questionário.

A pesquisa teve início após aprovação do Comitê de Ética em Pesquisa (CEP) institucional sob o número de parecer 2.465.427. As participantes foram convidadas pela pesquisadora por meio de convite divulgado e enviado pelas redes sociais (Facebook e Twitter) de coletivos feministas e LGBT, em que constava o link do questionário com informações a respeito da proposta do estudo, esclarecimentos quanto aos objetivos e metodologia, os riscos e os benefícios, a fim de que as mulheres julgassem a participação de forma voluntária. O questionário foi organizado no Google Docs e enviado de forma on-line para as participantes.

Depois de aceito o convite e respondida a primeira seção do questionário com o Termo de Consentimento Livre e Esclarecido (TCLE), as participantes tiveram acesso às demais seções e responderam, na íntegra, às questões solicitadas.

Para a coleta de dados foi utilizado o questionário adaptado de Pinto et al. (2005), anônimo, composto por perguntas obrigatórias e facultativas dividido em quatro domínios: dados pessoais, história ginecológica, conhecimento sobre o contágio por ISTs e conhecimento sobre métodos de prevenção de ISTs.

A análise dos dados foi realizada por meio da estatística descritiva das variáveis. Foram respondidos 119 questionários e selecionados, pela elegibilidade, 112, conforme os critérios apresentados no fluxograma dos critérios de inclusão e exclusão (Figura 1).

Editora Unijuí - Revista Contexto \& Saúde - ISSN 2176-7114 - v. 21, n. 43, jul./set. 2021 
Figura 1 - Fluxograma dos critérios de inclusão e exclusão

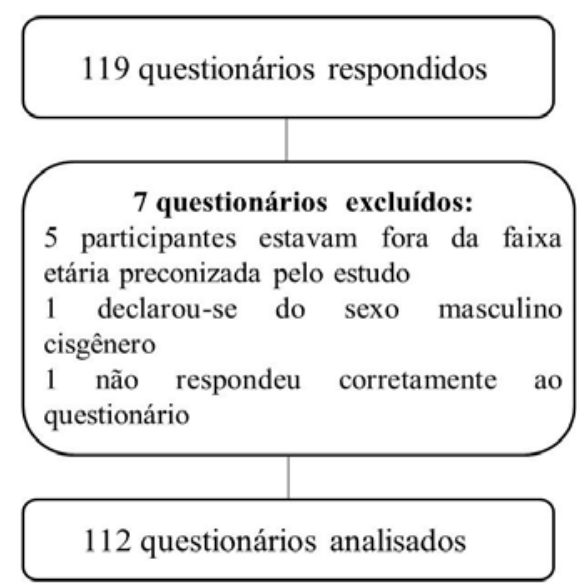

Fonte: Elaborado pelas autoras.

\section{RESULTADOS}

Participaram deste estudo 112 mulheres que responderam o questionário sobre o contágio por ISTs e conhecimento sobre métodos de prevenção de ISTs. As participantes tinham média de idade de $21,7 \pm 2,7$ anos. A Tabela 1 apresenta os dados de caracterização das mulheres jovens que fazem sexo com outras mulheres que, predominantemente, se autodeclararam lésbicas e com parceira fixa. Quanto ao acompanhamento ginecológico, $87,5 \%$ das mulheres realizam consultas ( $2,3 \pm 1,3$ consultas ao ano), mas somente $52,7 \%$ relatam já ter realizado o exame citopatológico.

Tabela 1 - Dados de caracterização das mulheres jovens, apresentados em porcentagem

\begin{tabular}{llll}
\hline Características & & N & \% \\
\hline Participantes & & 112 & $100 \%$ \\
Orientação Sexual & Bissexual & 44 & 39,3 \\
& Heterossexual & 09 & 8,00 \\
& Lésbica & 59 & 52,7 \\
Parceira Fixa & Sim & 60 & 53,6 \\
Já realizou sexo com homens & Não & 52 & 46,4 \\
& Sim & 69 & 61,6 \\
Realiza consulta ginecológica & Não & 43 & 38,4 \\
& Sim & 98 & 87,5 \\
Já realizou exame citopatológico & Não & 14 & 12,5 \\
& Sim & 59 & 52,7 \\
Já realizou testes de diagnóstico & Não & 53 & 47,3 \\
& Sífilis & 49 & 43,8 \\
& HIV & 59 & 52,7 \\
& Hepatite B & 54 & 48,2 \\
& Hepatite C & 53 & 47,3 \\
\hline
\end{tabular}

Fonte: Elaborado pelas autoras. 
A Tabela 2 apresenta a caracterização do conhecimento sobre métodos de prevenção de ISTs em MSM.

Tabela 2 - Compreensão sobre contágio por ISTs e métodos de prevenção em MSM, apresentada em porcentagem

\begin{tabular}{|c|c|c|c|}
\hline Prevenção de ISTs & & SIM (\%) & NÃO (\%) \\
\hline $\begin{array}{l}\text { Considera as práticas sexuais entre MSM } \\
\text { inseguras }\end{array}$ & & $98(87,5)$ & $14(12,5)$ \\
\hline $\begin{array}{l}\text { Relação sexual com homens apresenta maior } \\
\text { risco de } \\
\text { transmissão de ISTs }\end{array}$ & & $52(46,4)$ & $60(53,6)$ \\
\hline $\begin{array}{l}\text { Deixaria de ter relação sexual com mulheres } \\
\text { que se relacionam sexualmente com homens }\end{array}$ & & $6(5,4)$ & $\begin{array}{c}106 \\
(94,6)\end{array}$ \\
\hline $\begin{array}{l}\text { Conhece métodos de prevenção de ISTs para } \\
\text { MSM }\end{array}$ & & $61(54,5)$ & $51(45,5)$ \\
\hline \multirow[t]{2}{*}{$\begin{array}{l}\text { Usa métodos de prevenção de ISTs para } \\
\text { relações sexuais MSM }\end{array}$} & Sem acessórios & $10(8,9)$ & $\begin{array}{c}102 \\
(91,1)\end{array}$ \\
\hline & Com acessórios & $11(9,8)$ & $\begin{array}{c}101 \\
(90,2)\end{array}$ \\
\hline
\end{tabular}

Fonte: Elaborado pelas autoras.

Observa-se que a maioria das mulheres considera as práticas sexuais entre mulheres inseguras, ou seja, passíveis de transmissão de ISTs (87,5\%), e 54,5\% conhecem pelo menos um método de prevenção, porém apenas $8,9 \%$ usam algum tipo de barreira nas relações que não envolvem o uso de acessórios sexuais e 9,8\% utilizam nas relações que envolvem o uso desses acessórios.

Entre os principais motivos citados para a não adesão aos métodos de prevenção, estão: não ter conhecimento suficiente sobre a utilização correta e a falta de informação sobre a transmissão de ISTs entre mulheres $(27,6 \%)$, não usar acessórios sexuais na relação (22,4\%), ter uma parceira fixa em quem confia $(15,8 \%)$ ou considerar os métodos existentes desconfortáveis $(10,5 \%)$.

Em relação aos métodos preventivos utilizados pelas mulheres entrevistadas, $83,6 \%$ conhecem algum método para a prática de tribadismo (contato entre vulvas), 59\% para relações com uso de acessórios sexuais, 59\% para sexo anal, $19,7 \%$ para sexo vaginal e $34,4 \%$ para sexo oral. Entre os métodos citados estão: plástico filme ou preservativo feminino ou masculino aberto para o sexo oral (50\%), dedal/luva para a penetração $(24,5 \%)$, preservativo masculino ou feminino para sexo vaginal/anal $(63,3 \%)$, para o tribadismo $(18,4 \%)$ e nos acessórios sexuais $(36,7)$.

As características que as participantes julgam importantes constar no método preventivo de ISTs ideal para MSM, aumentando a adesão ao uso, são demonstradas na Tabela 3. Vale ressaltar que essa era uma pergunta aberta, de resposta pessoal e caráter não obrigatório, na qual elas poderiam responder mais de uma característica que julgassem importantes, caso desejassem. 
Tabela 3 - Características do método de prevenção de ISTs ideal de acordo com as mulheres jovens que fazem sexo com outras mulheres, em número absoluto e percentual

\begin{tabular}{lcc}
\hline Características & N & $\%$ \\
\hline Informação sobre o que previne e como usar & 23 & 25,8 \\
Praticidade & 21 & 23,6 \\
Valorizado e divulgado pela comunidade de MSM & 17 & 19,1 \\
Conforto & 11 & 12,4 \\
Informação sobre a transmissão de ISTs em MSM & 7 & 7,9 \\
Barato/Acessível/Fácil acesso & 5 & 5,6 \\
Especificidade para MSM & 5 & 5,6 \\
\hline
\end{tabular}

Fonte: Elaborado pelas autoras.

Observa-se que, entre os principais resultados, estão informações sobre a transmissão e prevenção de ISTs e o modo de uso do método preventivo, evidenciando a lacuna sobre educação sexual e práticas sexuais seguras deixadas pelos profissionais de saúde na população de MSM. Além disso, como não existe um método exclusivo para as MSM, mas há o preservativo para o sexo envolvendo homens, as mulheres precisam adaptar os métodos existentes, e conforme citado pelas entrevistas, acaba se tornando desconfortável (10,6\%) e difícil de usar $(6,6 \%)$, justificando a solicitação por um método específico para MSM (5,6\%).

\section{DISCUSSÃO}

Os resultados deste estudo demonstram que, na população MSM, a orientação sexual está relacionada à menor proteção contra ISTs. Apesar de a maioria das mulheres analisadas terem conhecimento sobre os métodos de proteção, menos de $10 \%$ fazem uso durante as relações sexuais. Isso se deve ao fato, principalmente, da falta de informações corretas sobre como usar os métodos preventivos ou por associarem o sexo entre mulheres com uma menor taxa de transmissão de ISTs. Estudos realizados na América Latina (SILBERMAN; BUEDO; BURGOS, 2016; SOUTO PEREIRA; SWAINSTON; BECKER, 2019), na América do Norte (MUZNY et al., 2013a) e na Europa (KOWALCZYK; NOWOSIELSKI, 2019) confirmam os achados desta pesquisa.

Alguns profissionais acham irrelevante questionar se a paciente teve relações com homens ou mulheres, pois o tratamento medicamentoso para a IST será o mesmo, independente do sexo da pessoa com quem ela se relacionou (SOUTO PEREIRA; SWAINSTON; BECKER, 2019). Então, raramente são discutidas alternativas de prevenção para o sexo entre mulheres, dificultando a educação sexual e o acesso à informação adequada (FUJII, 2019). Ao não orientarem e informarem adequadamente, tanto sobre as taxas de transmissão quanto sobre as maneiras como os métodos de proteção podem ser usados, é possível perceber a precariedade da atenção prestada às MSM pelos serviços de saúde brasileiros (RUFINO et al., 2018). 
Esse despreparo pode aparecer na recusa de discutir assuntos relacionados às práticas sexuais, no preconceito que a paciente fica sujeita a sofrer, na incapacidade de criar um ambiente acolhedor para a discussão da orientação sexual da mulher e na ignorância das especificidades existentes para cada grupo sexual, incluindo técnicas e estratégias de prevenção de ISTs (ALMEIDA, 2009).

Além disso, os profissionais pressupõem a heterossexualidade já oferecendo métodos de prevenção masculinos e anticoncepcionais, sem perguntar sobre a orientação sexual das pacientes (BRASIL, 2015). O relato da lesbianidade ou bissexualidade no momento da consulta está relacionado com a tentativa de direcionar a consulta para pautas que importam à mulher, desvencilhando-se da heteronormatividade (BARBOSA; FACCHINI, 2009).

Para que $\mathrm{o}$ atendimento integral aconteça é preciso entender quais as particularidades do grupo em questão, para que as orientações e cuidados sejam adequados com suas necessidades; assim, o treinamento dos profissionais da saúde sobre as práticas e necessidades de mulheres que fazem sexo com outras mulheres é crucial para o oferecimento de um serviço apropriado (BRASIL, 2015; LOGIE et al., 2015), porém, para que isso ocorra, é preciso uma mudança no modo de agir e de pensar dos profissionais de saúde (CARDOSO; FERRO, 2012).

A associação de contágio por ISTs por sexo com homens é muito comum entre MSM. A percepção é que homens transmitem mais facilmente os vetores das infecções, contagiando mulheres ao fazerem sexo desprotegido com elas, as quais, consequentemente, acabam transmitindo para suas parceiras femininas. Assim, as MSM e mulheres que fazem sexo com homens e mulheres (MSHM) seriam mais suscetíveis a infecções por ISTs por manterem relações de caráter heterossexual (MUZNY et al., 2013a). Em nossos achados, 46,4\% acreditam que MSHM apresentam maior risco de transmissão de ISTs para suas parceiras, porém apenas 5,4\% deixariam de ter esse tipo de relações.

O índice de mulheres que não usam métodos preventivos em relações monogâmicas é maior que o das que não estão em um relacionamento (ROWEN et al., 2013), em razão do equívoco de considerar uma relação homoafetiva-sexual monogâmica como segura (SILBERMAN; BUEDO; BURGOS, 2016). Mesmo em relações monogâmicas, porém, como há a troca de fluidos vaginais e corporais, é importante usar métodos preventivos para evitar o risco de transmissão de ISTs. Nesta pesquisa, um dos motivos apontados para justificar a falta de proteção nas relações sexuais é ter uma parceira fixa: 53,6\% das mulheres afirmaram estar em um relacionamento em média de 17,6 613,1 meses e é justamente por isso que $15,8 \%$ não se previnem.

A realização de testes rápidos para diagnósticos para ISTs é outro aspecto importante da saúde ginecológica de mulheres que, muitas vezes, é negligenciado pelos profissionais de saúde. As MSM, por não usarem proteção nas relações sexuais, são uma população de risco para contágio por ISTs, apesar de, muitas vezes, os profissionais não as verem como tal. Estudo africano (ZAIDI et al., 2016) realizado com mulheres que tiveram pelo menos uma parceira sexual nos últimos três anos, relata que a maioria das entrevistadas já realizou, pelo menos uma vez, o teste rápido para HIV; tal resultado é semelhante ao achado nesta pesquisa, em que $52,7 \%$ já foram testadas, porém, quando questionadas

Editora Unijuí - Revista Contexto \& Saúde - ISSN 2176-7114 - v. 21, n. 43, jul./set. 2021 
sobre a testagem para sífilis, hepatite B e C, a porcentagem diminui para $43,8 \%$, $48,2 \%$ e $47,3 \%$, respectivamente.

Quando perguntadas sobre o que as levou a realizar o teste, $80,3 \%$ responderam que foi por vontade própria e apenas 19,7\% que foi por solicitação médica. Pesquisa realizada na Argentina (SILBERMAN; BUEDO; BURGOS, 2016) com mulheres lésbicas e bissexuais maiores de 18 anos, apresenta dados semelhantes aos achados neste estudo. Como já observado, apesar de as MSM apresentarem práticas sexuais de risco para ISTs, elas ainda são vistas como uma população sem risco de contágio e, por isso, são pouco propensas a receber encaminhamentos por parte dos profissionais de saúde para a realização de testes rápidos (SCHICK et al., 2012).

Sobre a realização do exame citopatológico, também conhecido como Papanicolau, 52,7\% já o realizaram durante a consulta ginecológica. A maioria, $37,3 \%$, porém, realizou apenas uma vez. Estudo sul-americano demonstra que MSM recebem menos indicação para o exame preventivo (SILBERMAN; BUEDO; BURGOS, 2016). Essa falha na atenção à saúde de MSM torna-se ainda mais evidente, posto que a recomendação é de que o Papanicolau seja realizado anualmente, podendo o intervalo aumentar para três anos após dois testes consecutivos negativos para neoplasias e displasias (MS, 2016).

Em relação ao conhecimento sobre o método de prevenção, 54,5\% teve conhecimento sobre o método pela internet e apenas $9,8 \%$ pelo médico ginecologista, demonstrando o despreparo dos profissionais da saúde em prover educação sexual integralmente para as MSM. O alto índice de MSM que conheceram métodos pela internet também foi encontrado em um estudo argentino (SILBERMAN; BUEDO; BURGOS, 2016).

Quando perguntadas em quais práticas, atualmente, as mulheres fazem uso de proteção nas relações sem brinquedos sexuais, 11,1\% confirmaram usar proteção para o sexo oral, 77,8\% para o vaginal, 33,3\% para o anal e nenhuma afirmou usar algum método de barreira para a prática do tribadismo. Nas relações com acessórios sexual, 9,8\% confirmaram se proteger ao fazer uso desses na atividade sexual, com predominância do uso de camisinha masculina como método de barreira $88,89 \%$. Resultados alarmantes surgiram, também, em um estudo brasileiro (RUFINO et al., 2018) e um norte-americano (ROWEN et al., 2013), com semelhança nos resultados achados por este estudo no que se refere a não proteção no sexo oral e em relações com acessórios sexuais. É importante ressaltar que há riscos específicos de transmissão para cada prática sexual e, por isso, tem-se a necessidade de manter a proteção adequada em todas as relações sexuais (ROWEN et al., 2013).

O contágio por ISTs configura um problema de saúde pública e o atendimento não satisfatório às MSM fere o direito à saúde garantido por lei. Já há estudo que comprova que a taxa de contágio pode ser diminuída com ações de educação em saúde e intervenções públicas (LOGIE et al., 2015b), como campanhas de conscientização para os riscos de contágio por ISTs no sexo entre mulheres, de identificação dos sinais e sintomas das infecções mais comuns (LÚClO et al., 2019) e de incentivo às práticas sexuais seguras, por intermédio do ensino de meios de utilização dos métodos de barreiras adaptados.

Editora Unijuí - Revista Contexto \& Saúde - ISSN 2176-7114 - v. 21, n. 43, jul./set. 2021 
É necessária, também, a compreensão de que questionar as práticas e orientação sexual das mulheres atendidas auxilia no acolhimento e na humanização da assistência das MSM, além da identificação das vulnerabilidades e dificuldades encontradas por elas no acesso aos serviços de saúde (RUFINO et al., 2018).

Como limitações deste estudo encontra-se a homogeneidade da amostra, que foi totalmente recrutada por meio das redes sociais e tornava obrigatório o acesso à internet para participação na pesquisa, o uso de um questionário não validado para coleta de dados e a não investigação da prevalência de ISTs nas mulheres da amostra. Nenhuma dessas limitações, contudo, afetou a importância do estudo.

\section{CONCLUSÃO}

As MSM têm conhecimento sobre os riscos de contágio por ISTs a que são expostas ao realizarem sexo sem proteção, porém, ainda assim, não têm o costume de usar métodos preventivos. Isso pode ser explicado pela lacuna de conhecimento que os profissionais de saúde apresentam sobre a saúde e práticas sexuais desse grupo. Pouquíssimas mulheres tiveram acesso a informações sobre prevenção de ISTs por meio dos profissionais de saúde, reforçando a importância da necessidade de capacitação para que as MSM possam ter seu direito à saúde cumprido.

A utilização de métodos de barreiras não é recorrente nas relações, pois muitas os consideram pouco usuais e práticos, uma vez que não existe um específico para MSM e, assim, realizam adaptações nos já existentes como forma de se prevenir. Além disso, poucas mulheres receberam orientações sobre a prevenção de ISTs por médicos ginecologistas ou profissionais da saúde, e recorrem à busca por informações sobre métodos preventivos na internet.

A prevenção de ISTs ainda é um tema distante para as MSM, sendo imprescindível que os profissionais de saúde as vejam como um grupo de risco para contágio por ISTs, informando-as corretamente sobre métodos preventivos e incluindo-as nos exames de rastreamento recomendados. Por último, conclui-se que, para a atenção ocorrer de forma integral e satisfatória, é preciso que mais estudos sejam realizados na área de saúde sexual e ginecológica das MSM.

\section{REFERÊNCIAS}

ALMEIDA, G. Argumentos em torno da possibilidade de infecção por DST e Aids entre mulheres que se autodefinem como lésbicas. Physis Revista de Saúde Coletiva, v. 19, n. 2, p. 301-331, 2009.

BARBOSA, R. M.; FACCHINI, R. Acesso a cuidados relativos à saúde sexual entre mulheres que fazem sexo com mulheres em São Paulo, Brasil. Cadernos de Saúde Pública, v. 25, n. 2, p. S291-300, 2009.

BRASIL. Senado Federal. 2013. Estatuto da juventude: atos internacionais e normas correlatas. Disponível em: https://www2.senado.leg.br/bdsf/bitstream/handle/ id/509232/001032616.pdf. Acesso em: 27 maio 2018.

Editora Unijuí - Revista Contexto \& Saúde - ISSN 2176-7114 - v. 21, n. 43, jul./set. 2021 
BRASIL. Cartilha: campanha para lésbicas e bissexuais. Brasília, DF: Ministério da Saúde, 2015.

CARDOSO, M. R.; FERRO, L. F. Saúde e população LGBT: demandas e especificidades em questão. Psicologia: ciência e profissão, v. 32, n. 3, p. 552-563, 2012.

DALY, F.; SPICER, N.; WILLAN, S. Sexual rights but not the right to health? Lesbian and bisexual women in South Africa's National Strategic Plans on HIV and STIs. Reproductive Health Matters, v. 24, n. 47, p. 185-194, May. 2016.

FUJI, H. Sexual norms for lesbian and bisexual women in a culture where lesbianism is not acceptable enough: the japanese survey about sexual behaviors, stis preventive behaviors, and the value of sexual relations. Journal of Homosexuality, v. 66, n. 3, p. 407420, Feb. 2019.

KOWALCZYK, R.; NOWOSIELSKI, K. Impact of social factors and sexual behaviors on the knowledge of sexually transmitted infections among women who have sex with women/ women who have sex with women and men. International Journal of STD and Aids, v. 30, n. 2, p. 163-172, Feb. 2019.

LOGIE, C. H. (Where) do queer women belong? Theorizing intersectional and compulsory heterosexism in HIV research. Critical Public Health, v. 25, n. 5, p. 527-538, 2015.

LOGIE, C. H. et al. A Pilot Study of a Group-Based HIV and STI Prevention Intervention for Lesbian, Bisexual, Queer, and Other Women Who Have Sex with Women in Canada. AIDS Patient Care and STDs, v. 29, n. 6, p. 321-328, abr. 2015.

LÚCIO, F. P. DA S. et al. Saúde sexual da mulher lésbica e/ou bissexual: especificidades para o cuidado à saúde e educação sexual. Revista Ibero-Americana de Estudos em Educação, Araraquara, v. 14, n. n. esp. 2, p. 1.465-1.479, jul. 2019.

MS. Ministério da Saúde. Instituto Nacional de Câncer José Alencar Gomes da Silva (Inca). 2016. Diretrizes brasileiras para o rastreamento do câncer do colo do útero. Disponível em: https://www.inca.gov.br/sites/ufu.sti.inca.local/files//media/document//diretrizesparaorastreamentodocancerdocolodoutero_2016_corrigido.pdf. Disponível em: 13 ago. 2019.

MUZNY, C. A. et al. Sexual behaviors, perception of sexually transmitted infection risk, and practice of safe sex among southern African American women who have sex with women. Sex Transm Dis, v. 40, n. 5, p. 395-400, May. 2013 a.

MUZNY, C. A. et al. Misperceptions regarding protective barrier method use for safer sex among African-American women who have sex with women. Sex Health, v. 10, n. 2, p. 138-141, 2013b.

OLIVEIRA, A. D. DA S.; NERY, I. S. Mulheres lésbicas no contexto do HIV/AIDS: revisão integrativa. Revista de Enfermagem Ufpe on-line, Recife, v. 10, n. 8, p. 3.090-3.100, ago. 2016.

PINTO, V. et al. Sexually transmitted disease/HIV risk behaviour among women who have sex with women. Aids, v. 19, n. 4, p. S64-69, 2005.

ROWEN, T. S. et al. Use of barrier protection for sexual activity among women who have sex with women. International Journal of Gynecology and Obstetrics, v. 120, n. 1, p. 4245, Jan. 2013.

RUFINO, A. C. et al. Práticas sexuais e cuidados em saúde de mulheres que fazem sexo com mulheres: 2013-2014. Epidemiologia e Serviços de Saúde: Revista do Sistema Único de Saúde do Brasil [on-line], Brasília, v. 27, n. 4, p. e2017499, nov. 2018.

SCHICK, V. et al. Sexual behaviour and risk reduction strategies among a multinational sample of women who have sex with women. Sex Transm Infect, v. 88, n. 6, p. 407-412, Oct. 2012.

SILBERMAN, P.; BUEDO, P. E.; BURGOS, L. M. Barreras en la atención de la salud sexual en Argentina: percepción de las mujeres que tienen sexo con mujeres. Revista de Salud Pública, v. 18, n. 1, p. 1-12, feb. 2016.

SOUTO PEREIRA, S.; SWAINSTON, K.; BECKER, S. The discursive construction of low-risk to sexually transmitted diseases between women who are sexually active with women. Culture, Health and Sexuality, v. 21, n. 11, p. 1.309-1.321, Nov. 2019. 
TAT, S. A.; MARRAZZO, J. M.; GRAHAM, S. M. Women who have sex with women living in low- and middle-income countries: A systematic review of sexual health and risk behaviors. LGBT Health, v. 2, n. 2, p. 91-104, Jun. 2015.

VARELA, A. I. S. et al. Comprometimento da sexualidade de mulheres com câncer de mama. Enfermagem em Foco, v. 8, n. 1, p. 67-71, abr. 2017.

ZAIDI, S. S. et al. Women who have sex with women in Kenya and their sexual and reproductive health. LGBT Health, v. 3, n. 2, p. 139-145, Apr. 2016.

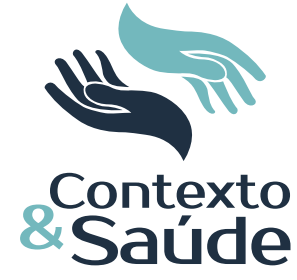

